\title{
Magnetic and Structural Properties of FeCr Alloys
}

\author{
A. Froideval, ${ }^{1}$ R. Iglesias, ${ }^{1}$ M. Samaras, ${ }^{1}$ S. Schuppler, ${ }^{2}$ P. Nagel, ${ }^{2}$ D. Grolimund,,${ }^{1,3}$ M. Victoria, ${ }^{1,4,5}$ and W. Hoffelner ${ }^{1}$ \\ ${ }^{1}$ Paul Scherrer Institute, Nuclear Energy and Safety, 5232 Villigen PSI, Switzerland \\ ${ }^{2}$ Forschungszentrum Karlsruhe, Institut für Festkörperphysik, 76021 Karlsruhe, Germany \\ ${ }^{3}$ Paul Scherrer Institute, Swiss Light Source, 5232 Villigen PSI, Switzerland \\ ${ }^{4}$ Lawrence Livermore National Laboratory, Chemistry and Materials Science, Livermore, California 94550, USA \\ ${ }^{5}$ Polytechnic University of Madrid, Institute of Nuclear Fusion, 28006 Madrid, Spain
}

(Received 25 May 2007; published 4 December 2007)

\begin{abstract}
Synergistic synchrotron $\mathrm{x}$-ray absorption experiments using imaging magnetic microspectroscopy, $\mathrm{x}$-ray magnetic circular dichroism, and ab initio calculations on $\mathrm{FeCr}$ alloys reveal that the $\mathrm{Cr}$ content strongly influences the ferromagnetic microstructure and the Fe magnetic moments. The $\mathrm{Cr}$ local structure resolved by extended x-ray absorption fine structure (EXAFS) is also found to be affected by the alloy's composition. Both EXAFS and ab initio calculations show a change in the $\mathrm{Cr}$ local atomic structure above 10 at.\% $\mathrm{Cr}$ content from the distance contraction of the first two coordination shells around the $\mathrm{Cr}$ absorbing atom. These results indicate the strong dependence of magnetic and structural properties of these alloys on $\mathrm{Cr}$ concentration.
\end{abstract}

DOI: 10.1103/PhysRevLett.99.237201

PACS numbers: 75.50.Bb, 71.15.Mb, 71.20.Be, 78.70.Dm

Industrial steels, whose chemical compositions are based on an $\mathrm{FeCr}$ matrix with $\mathrm{Cr}$ concentrations ([Cr]) ranging from 2-20 atomic percentage (at. \%), are possible candidates foreseen for the design of structural components in advanced nuclear energy installations such as Generation IV and fusion reactors. Until recently, modeling of such alloys has been pursued by excluding magnetism (due to the additional complications it produces) [1,2], regardless of the knowledge that magnetism stabilizes body centered cubic (bcc) $\mathrm{Fe}$ [3], that magnetic effects have been observed to cause a $\mathrm{Cr}$ content dependent heterogeneous magnetic structure in $\mathrm{FeCr}$ [4], and that a crossover from short range order at low $[\mathrm{Cr}]$ to clustering at high $[\mathrm{Cr}]$ exists at approximately 10 at. \% $\mathrm{Cr}[5,6]$. Moreover, recent theoretical calculations predict a negative sign of the heat of formation of $\mathrm{FeCr}$ alloys with [Cr] lower than 10-12 at. $\%[7,8]$ emphasizing the crucial role played by magnetism in the stability of the $\mathrm{FeCr}$ system. A complete understanding of the magnetic properties of $\mathrm{FeCr}$ alloys is thus needed to meet the challenge of developing advanced nuclear materials resistant to high radiation doses and temperatures. To date, no study combining both elementspecific spectroscopy and ab initio calculations has been devoted to resolve this issue. This Letter presents an approach based on the complementary use of photoemission electron microscopy (PEEM) with circularly polarized soft $\mathrm{x}$-rays and $\mathrm{x}$-ray magnetic circular dichroism (XMCD), coupled with $a b$ initio density functional theory investigations, to assess the influence of alloy composition on the magnetic microstructure of the $\mathrm{FeCr}$ system. Additionally, extended x-ray absorption fine structure (EXAFS) measurements on $\mathrm{FeCr}$ alloys have been performed and compared with $a b$ initio calculations to study the $\mathrm{Cr}$ local structure in the $\mathrm{FeCr}$ alloys with varying [Cr].

$A b$ initio calculations have been performed on bcc Fe and an $\mathrm{Fe}-12.5 \% \mathrm{Cr}$ alloy using the FLAPW (Full-potential
Linearized Augmented Plane Wave) method implemented in the WIEN2k code [9]. The exchange correlation potential assumed was the PBE (Perdew-Burke-Ernzerhof, [10]) parameterization of the GGA (Generalized Gradient Approximation). Self-consistent FLAPW calculations were initially performed for pure bcc Fe in order to check the reliability of our computational procedure and concurrently provide a reference system [11]. In contrast to results obtained from LSDA (Local Spin Density Approximation) [12], the use of GGA leads to the correct ferromagnetic ground state, with a lattice parameter of $2.83 \AA$, an $\mathrm{Fe}$ magnetic moment of $2.17 \mu_{B}$, orbital, $m_{L}$, and spin, $m_{S}$, magnetic moments of 0.078 and 2.248 , respectively, and their ratio $m_{L} / m_{S}$ of 0.035 [11], all of which compare well with the experimental results for bcc $\mathrm{Fe}[13,14]$. Subsequently, calculations on the $\mathrm{FeCr}$ alloys were performed. Here, the description is focused on the $\mathrm{Fe}$ $12.5 \% \mathrm{Cr}$ system. A full relaxation of the lattice constant, the crystal structure, and the atomic positions was carried out for the entire set of $\mathrm{Fe}-12.5 \% \mathrm{Cr}$ alloy structures studied, simulated by means of the supercell (SC) approach. The SCs contain $8(2 a \times 2 a \times a)$ atoms, with one $\mathrm{Cr}$ atom substituting one Fe atom, and $16(2 a \times 2 a \times$ $2 a$ ) atoms, with two $\mathrm{Cr}$ atoms substituting two $\mathrm{Fe}$ atoms. The radius of the muffin-tin spheres, $R_{\mathrm{MT}}$, for both the Fe and $\mathrm{Cr}$ atoms, was chosen to be 2.1 a.u. for all cases. The plane wave cutoff was set to 9.0 , and the number of $k$ points in the irreducible wedge of the Brillouin zone was 150 for the 8 -atom and 84 for the 16 -atom SCs, respectively. The relaxation takes place in two steps. In the low $[\mathrm{Cr}]$ region of interest, the theoretical pure bcc Fe lattice constant ( $a=$ $2.83 \AA$ ) can be taken as the starting point for a calculation in which energy and charge were fully converged. As the forces present in the system were observed to be large (about $5.0 \mathrm{~m} \mathrm{Ry} / \mathrm{a} . u$.) due to the presence of two distinct species of atoms in the structure, optimization of the 
internal atomic positions was performed to ensure energy, charge, and force convergence. The equilibrium volume, energy, bulk modulus, and its first derivative with regards to pressure were determined from fits to the Murnaghan's equation of state [15]. Together with the optimized internal positions for that volume, a theoretical prediction of the structure of the material is given. All the above calculations were run without including spin-orbit (SO) coupling. Subsequently, SO coupling was added using the second variational step approach [16] with the magnetization confined to the (001) or $z$ direction. It is well known that the energy density functional together with SO coupling must contain terms which depend directly on the orbital moment. When SO is not included, a considerable underestimation of this quantity is found for $3 d$ transition metals [17]. The orbital momentum may be enhanced by the inclusion of an orbital polarization (OP) term in the Hamiltonian [18] in an attempt to account for the second Hund's rule [19], which is again treated within the second variational method using the same $k$-point set as for the SO calculation. The absolute $m_{L}, m_{S}$ moments and their ratio $m_{L} / m_{S}$ for $\mathrm{Fe}$ in the 8-and the 16-atom $\mathrm{Fe}-12.5 \% \mathrm{Cr}$ alloy SCs were calculated after averaging over the inequivalent atoms. Several configurations with different positions of the $\mathrm{Cr}$ substituting atoms were tested for both SCs. From these, only the structures corresponding to the energy minima have been considered here. For the 8-atom SC, the energy minimum is obtained when the substituting $\mathrm{Cr}$ atom is at $(0.5,0,0)$ and $a=2.846 \AA$. It should be noted, however, that the value of the energy is only $0.5 \mathrm{mRy}$ lower than when the $\mathrm{Cr}$ sits in another position, such as $(0.75$, $0.75,0.5$ ), indicating a similar stability of the various configurations. The most stable configuration of the 16atom SC is found when the $\mathrm{Cr}$ atom is placed as a substitutional atom located at $(0,0,0)$, and the second substituted $\mathrm{Cr}$ is placed either at $(0.5,0.5,0),(0.5,0,0.5)$, or $(0,0.5$, 0.5 ), which are completely equivalent from a structural point of view. The optimized lattice parameter is found to be $2.845 \AA$ in all three cases and is only slightly smaller than for the 8-atom SC calculation. Comparing the results of the two SCs reveals that there is a size effect resulting in small reductions of up to $4 \%$ in the Fe magnetic moments and the $m_{L} / m_{S}$ ratio when going from the 8-atom to the 16atom SC. The average total magnetic moment for the two SCs $\left(1.853 \mu_{B}\right)$ favorably compares to the bulk magnetization measurement $\left(1.916 \mu_{B}\right)$ [20] and is lower than that of bcc Fe $\left(2.17 \mu_{B}\right)$ as expected. In contrast, from the data presented in Table I, the spin magnetic moment is enhanced in the $\mathrm{Fe}-12.5 \% \mathrm{Cr}$ alloy. These new results prove that the presence of $\mathrm{Cr}$ greatly influences the magnetic properties of $\mathrm{FeCr}$ alloys.

To validate these calculations and obtain further insight into the role of magnetism, XMCD and EXAFS experiments were undertaken. Button melts of $\mathrm{FeCr}$ alloys were prepared by arc-melting the stoichiometric proportions of
TABLE I. Bulk and surface Fe $m_{L}, m_{S}$, and $m_{L} / m_{S}$ values for bcc $\mathrm{Fe}$ and an $\mathrm{Fe}-12 . x \% \mathrm{Cr}$ alloy with $x=5$, as computed, and with $x=7$, as estimated from XMCD experiments.

\begin{tabular}{lcccccc}
\hline \hline & \multicolumn{3}{c}{ Bulk Fe atoms } & \multicolumn{2}{c}{ Surface Fe atoms } \\
\hline & $m_{L} / m_{S}$ & $m_{L}$ & $m_{S}$ & \multicolumn{2}{c}{$m_{L} / m_{S}$} \\
& theory & $\mathrm{XMCD}$ & theory & theory & $\mathrm{XMCD}$ \\
$\mathrm{Fe}$ & 0.035 & $0.043^{\mathrm{a}}$ & 0.078 & 2.248 & 0.050 & 0.060 \\
$\mathrm{Fe} 12 . x \% \mathrm{Cr}$ & 0.027 & & 0.063 & 2.319 & & 0.037 \\
\hline \hline
\end{tabular}

${ }^{\mathrm{a}}$ From Ref. [14].

99.995\% pure metallic $\mathrm{Fe}$ and $99.999 \%$ pure metallic $\mathrm{Cr}$ under an argon atmosphere, with previous vacuum purging. The melting was performed several times to ensure compositional homogeneity. A thermal treatment which consists of $1 \mathrm{~h}$ at $950^{\circ} \mathrm{C}$, followed by an annealing in the furnace at $790^{\circ} \mathrm{C}$ under $8 \times 10^{-7}$ mbar pressure, completed the preparation. Prior to the measurements, the button melts were cut to flat samples of $7 \times 7 \mathrm{~mm}^{2}$ surface area and $1 \mathrm{~mm}$ thickness, polished down to $250 \mathrm{~nm}$, and ultrasonically cleaned in order to remove all traces of silica particles used at the last stages of polishing. The microstructure of the $\mathrm{FeCr}$ alloys investigated by Scanning Electron Microscopy (SEM) was found to be homogeneous. The composition of the $\mathrm{FeCr}$ alloys was determined by Inductively Coupled Plasma-Atomic Emission Spectrometry (ICP-AES). The $\mathrm{Cr}$ content of the alloys given in atomic percent was measured to be $6.2 \pm 0.3$ at. $\%$ and $12.7 \pm 0.2$ at. $\%$, and the total concentration of impurities was less than 0.05 at.\%. The element-resolved ferromagnetic microstructure of the two $\mathrm{FeCr}$ alloys and of the 99.995\% pure metallic Fe reference sample were also investigated by means of imaging magnetic microspectroscopy [21], which combines PEEM and XMCD. Prior to the measurements, in situ surface cleaning by low-energy Ar-sputtering was conducted on all samples. The PEEMXMCD measurements were performed at the Fe $L_{2,3}$ absorption edges using left- and right-handed circularly polarized synchrotron radiation at the WERA (WEichRoentgen-Analytik-Anlage) beam line in ANKA (ANgstroemquelle KArlsruhe) synchrotron light source (Karlsruhe, Germany). The degree of circular polarization and the angle between the incoming beam and the sample surface were set to $75 \%$ and $25^{\circ}$, respectively. A Focus PEEM was used to record the PEEM images of the ferromagnetic domains in zero magnetic field at room temperature. For the required spectral information, image stacks (consisting of typically 166 images) were taken for photon energies between 690 and $750 \mathrm{eV}$, covering the $\mathrm{Fe} L_{3}$ and $L_{2}$ edges, and for both left- and right-handed circular polarization. Two types of domains (bright and dark) having opposite magnetization were observed. Taking the difference of microspectra averaged over each type of domain results in a spectrum proportional to the XMCD signal [22]. While we did not attempt to determine the 

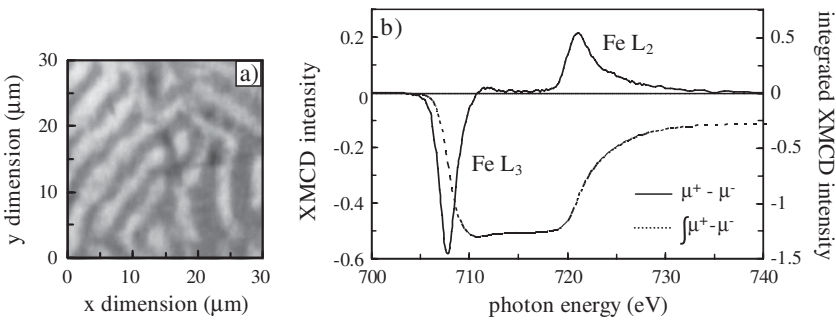

FIG. 1. (Micro-)spectroscopic results of bcc Fe: (a) $(30 \times$ 30) $\mu \mathrm{m}^{2}$ PEEM image recorded at the Fe $L_{3}$ edge, and (b) normalized Fe $L_{2,3}$ edges XMCD spectrum (solid line) and its integration (dotted line).

absolute Fe $m_{L}$ or $m_{S}$ values, their ratio was readily determined by integrating the XMCD signal according to the sum rules [14]. The experimental uncertainty of the resulting $m_{L} / m_{S}$ values was $3 \%$.

Figure 1 shows the PEEM image and the spectroscopic data of the Fe reference sample. The domain structure consists of an irregular sawtooth pattern, with the two types of domains clearly visible [Fig. 1(a)]. Dichroism is visible on the XMCD spectrum [Fig. 1(b)]. The value of the $m_{L} / m_{S}$ ratio for the $\mathrm{Fe}$ surface atoms, which is directly estimated from the integrated XMCD signal, is 0.060 (Table I) and is close to our theoretical value of 0.050 determined for an $\mathrm{Fe}$ surface in slab geometry using GGA [11], as well as to calculations that used LSDA where a ratio of 0.063 was obtained [23]. We thus demonstrate a clear concordance of the experimental and theoretical approaches to study the Fe surface magnetic moments in bcc Fe. Moreover, our results provide experimental evidence of the enhancement of the $m_{L} / m_{S}$ value of bcc Fe at the surface relative to the bulk, which we calculated as 0.035 and which was experimentally determined as 0.038 [13] and 0.043 [14].

The PEEM images and the spectroscopic data of the $\mathrm{Fe}-6.2 \% \mathrm{Cr}$ and $\mathrm{Fe}-12.7 \% \mathrm{Cr}$ alloys are presented in Fig. 2. The compositional difference of the alloys induces different ferromagnetic domain structures: a change of pattern from regular column [Fig. 2(a)] to sawtooth [Fig. 2(b)] is produced by increasing [Cr]. This change of the magnetic microstructure of the alloys has a direct influence on the XAS and the XMCD spectra shown in Figs. 2(c)-2(f), and consequently on the values of the $m_{L} / m_{S}$ ratio estimated from the integrated XMCD signals. An increase of [Cr] in the alloys from 6.2 at.\% to 12.7 at.\% induces an increase of the values of the $m_{L} / m_{S}$ ratio at the surface from 0.029 to 0.037 . The alloy value of the magnetic moment ratio is lower than the ratio determined for bcc Fe, namely, 0.060 (Table I), suggesting an influence of [Cr] on the magnetic properties at the $\mathrm{FeCr}$ surface. It is also interesting to note that the estimated higher $m_{L} / m_{S}$ value of the $\mathrm{Fe}-12.7 \% \mathrm{Cr}$ alloy equal to 0.037 is concomitant with the $\mathrm{Cr}$ segregation observed at the surface of a high-purity $\mathrm{Fe}-13.5 \% \mathrm{Cr}$ alloy
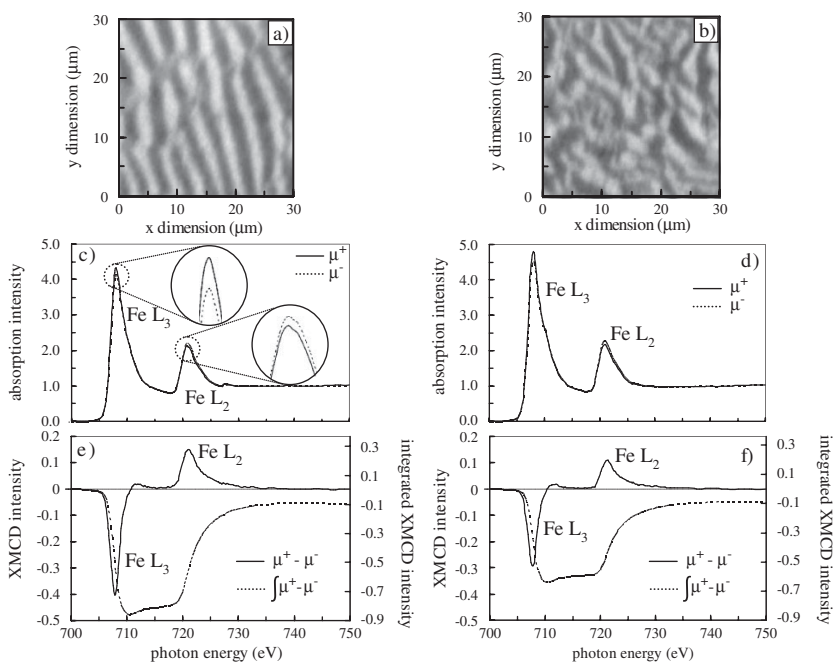

FIG. 2. (Micro-)spectroscopic results of the Fe-6.2\% Cr [left panel, (a, c, and e)] and Fe-12.7\% Cr [right panel, (b, d, and f)] alloys. (a-b) PEEM images recorded at the Fe $L_{3}$ edge, (c-d) normalized XAS spectra at the $\mathrm{Fe} L_{2,3}$ edges for positive ( $\mu^{+}$, dotted line) and negative $\left(\mu^{-}\right.$, solid line) helicities and (e-f) corresponding XMCD spectra $\left(\mu^{+}-\mu^{-}\right)$and their integrations $\left(\int \mu^{+}-\mu^{-}\right)$determined from the absorption spectra of panels (c) and (d), respectively.

[24]. The experiments clearly demonstrate the [Cr] dependence of the surface magnetic moments in $\mathrm{FeCr}$ alloys.

To understand the interplay between structure and magnetism, $\mathrm{Cr} K$ edge EXAFS measurements of the arc-melted $\mathrm{FeCr}$ alloys containing 6.2 at. \% and 12.7 at. \% $\mathrm{Cr}$ were performed at the microXAS beam line of the Swiss Light Source (SLS). Data were collected in fluorescence mode at room temperature. The Athena and Artemis packages which are part of the Ifeffit program [25] were used to process and to fit the EXAFS data, respectively. The evolution of the magnetic moments was correlated to the structural environments of the $\mathrm{Cr}$ atoms in the two binary alloys to study the dependence on $[\mathrm{Cr}]$. Figure 3 compares the experimental and Fourier transform fits of the EXAFS

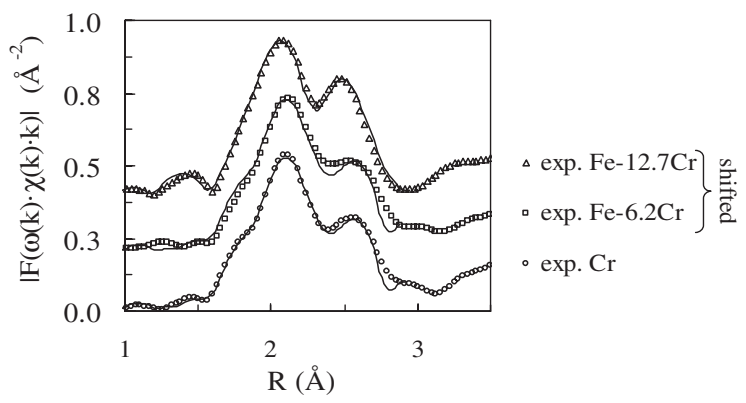

FIG. 3. Experimental (open symbols) and fit (solid line) of the Fourier transforms of the $\mathrm{Cr} K$ edge EXAFS data recorded on pure $\mathrm{Cr}, \mathrm{Fe}-6.2 \% \mathrm{Cr}$, and $\mathrm{Fe}-12.7 \% \mathrm{Cr}$ samples. Each alloy experimental curve and its associated fit are incrementally shifted by $0.2 \AA^{-2}$ for better visibility. 
TABLE II. Comparison of the interatomic distance $R$ (in $\AA$ ) from the $\mathrm{Cr}$ absorbing atom to its first $\left(A_{1}\right)$ and second $\left(A_{2}\right)$ neighbor atoms determined by fitting the first two shells of the Fourier transforms of EXAFS data recorded on pure $\mathrm{Cr}, \mathrm{Fe}-$ $6.2 \% \mathrm{Cr}$ and $\mathrm{Fe}-12.7 \% \mathrm{Cr}$ samples and by using FLAPW-GGA calculations for bec $\mathrm{Cr}$ and an $\mathrm{Fe}-12.5 \% \mathrm{Cr}$ alloy.

\begin{tabular}{|c|c|c|c|c|c|}
\hline $\begin{array}{l}\text { Sample } \\
\text { Distance }\end{array}$ & $\begin{array}{l}\mathrm{Cr} \\
\text { theory }\end{array}$ & $\begin{array}{c}\mathrm{Cr} \\
\text { EXAFS }\end{array}$ & $\begin{array}{c}\mathrm{Fe}-6.2 \% \mathrm{Cr} \\
\text { EXAFS }\end{array}$ & $\begin{array}{c}\mathrm{Fe}-12.5 \% \mathrm{Cr} \\
\text { theory }\end{array}$ & $\begin{array}{c}\mathrm{Fe}-12.7 \% \mathrm{Cr} \\
\text { EXAFS }\end{array}$ \\
\hline$R_{\mathrm{Cr}-A_{1}}$ & 2.495 & 2.49 & 2.48 & 2.464 & 2.46 \\
\hline$R_{\mathrm{Cr}-\mathrm{A}_{2}}$ & 2.881 & 2.88 & 2.88 & 2.845 & 2.82 \\
\hline
\end{tabular}

data recorded on the pure $\mathrm{Cr}$ reference, the $\mathrm{Fe}-6.2 \% \mathrm{Cr}$, and the $\mathrm{Fe}-12.7 \% \mathrm{Cr}$ samples. The average distances from the $\mathrm{Cr}$ absorbing atom to its first and second neighbors are listed in Table II. Excellent agreement is obtained between this EXAFS study, the theoretical calculations performed in this work and published crystallographic data for a pure Cr sample [26]. An interesting EXAFS result is that there is a change of the spatial distribution of the first and the second coordination shells around the $\mathrm{Cr}$ absorbing atom in $\mathrm{Fe}-6.2 \% \mathrm{Cr}$ and $\mathrm{Fe}-12.7 \% \mathrm{Cr}$. The $\mathrm{Cr}$ structural environment is thus affected by the $\mathrm{Cr}$ content of the alloys as well as by the relative positions inside the structure of the $\mathrm{Cr}$ atoms themselves. The position of the coordination shells within $4 \AA$ around the $\mathrm{Cr}$ is similar in the pure $\mathrm{Cr}$ reference sample and in the $\mathrm{Fe}-6.2 \% \mathrm{Cr}$ alloy, while the $\mathrm{Fe}-12.7 \% \mathrm{Cr}$ alloy exhibits a distance contraction of its first two coordination shells around the $\mathrm{Cr}$, in very good agreement with the results of the $a b$ initio calculations. Such a structural variation of the $\mathrm{Fe}-12.7 \% \mathrm{Cr}$ alloy compares well with previous anomalous $\mathrm{x}$-ray diffraction investigations of a Fe-47.2\% Cr alloy performed by Reinhard et al. [27] and evidences a clear influence of the $\mathrm{Cr}$ content of the alloy onto the $\mathrm{Cr}$ local atomic structure.

In conclusion, the combination of PEEM-XMCD, EXAFS and $a b$ initio FLAPW calculations on $\mathrm{FeCr}$ alloys proves that the magnetic and structural properties of $\mathrm{FeCr}$ alloys change with $[\mathrm{Cr}]$. The distance contraction of the two first shells around the $\mathrm{Cr}$ atom in the $\mathrm{Fe}-12.7 \% \mathrm{Cr}$ alloy is concomitant with the increase in the Fe magnetic moments. These results indicate the crucial effect that magnetism has on the structure of $\mathrm{FeCr}$ alloys and the necessity to include magnetism at the microscopic level to construct a robust modelling and experimental scheme for the design of future $\mathrm{FeCr}$-based steels.

We are grateful to F. Nolting for valuable discussions, A. Bullemer, M. Niffenegger, and U. Tschanz for sample preparation, M. Merz and A. Samartsev for their support during the PEEM measurement campaign, as well as C. Redaelli for computing support and the Swiss National
Supercomputing Centre (CSCS) for extensive use of their facilities. The PEEM was provided by FOCUS $\mathrm{GmbH}$ within their cooperation with the Institut für Festkörperphysik (IFP, Karlsruhe). We also acknowledge SLS and ANKA for the provision of beamtime.

[1] M. Samaras, W. Hoffelner, and M. Victoria, J. Nucl. Mater. 371, 28 (2007).

[2] M. Samaras, W. Hoffelner, C.-C. Fu, M. Guttmann, and R. E. Stoller, in Proceedings of ICAPP 2007 and Revue Générale Nucléaire (SFEN, France, 2007).

[3] H. Hasegawa and D. G. Pettifor, Phys. Rev. Lett. 50, 130 (1983).

[4] C. G. Shull and M. K. Wilkinson, Phys. Rev. 97, 304 (1955).

[5] I. Mirebeau, M. Hennion, and G. Parette, Phys. Rev. Lett. 53, 687 (1984).

[6] M. Y. Lavrentiev et al., Phys. Rev. B 75, 014208 (2007).

[7] P. Olsson, I. A. Abrikosov, and J. Wallenius, Phys. Rev. B 73, 104416 (2006).

[8] T. P. C. Klaver, R. Drautz, and M. W. Finnis, Phys. Rev. B 74, 094435 (2006).

[9] P. Blaha et al., WIEN2k, An Augmented Plane Wave + Local Orbitals Program for Calculating Crystal Properties (Techn. University, Vienna, Austria, 2001).

[10] J. P. Perdew, K. Burke, and M. Ernzerhof, Phys. Rev. Lett. 77, 3865 (1996).

[11] R. Iglesias et al. (to be published).

[12] J. Kübler, Theory of Itinerant Electron Magnetism (Clarendon, Oxford, 2000).

[13] H. P. J. Wijn, in Magnetic Properties of $3 d$, $4 d$ and $5 d$ Elements, Alloys and Compounds (Springer, Berlin, 1997).

[14] C. T. Chen et al., Phys. Rev. Lett. 75, 152 (1995).

[15] F. D. Murnaghan, Proc. Natl. Acad. Sci. U.S.A. 30, 244 (1944).

[16] D. D. Koelling and B. N. Harmon, J. Phys. C 10, 3107 (1977).

[17] P. Söderlind et al., Phys. Rev. B 45, 12911 (1992).

[18] O. Eriksson, B. Johansson, and M.S.S. Brooks, J. Phys. Condens. Matter 1, 4005 (1989).

[19] G. Racah, Phys. Rev. 62, 438 (1942).

[20] A. T. Aldred, Phys. Rev. B 14, 219 (1976).

[21] H. Hopster and H.P. Oepen, Magnetic Microscopy of Nanostructures (Springer, Berlin, 2005).

[22] A. Froideval et al. (to be published).

[23] O. Hjortstam et al., Phys. Rev. B 53, 9204 (1996).

[24] S. Suzuki et al., Appl. Surf. Sci. 103, 495 (1996).

[25] B. Ravel and M. Newville, J. Synchrotron Radiat. 12, 537 (2005).

[26] M.E. Straumanis and C. C. Weng, Acta Crystallogr. 8, 367 (1955).

[27] L. Reinhard et al., Phys. Rev. B 45, 2662 (1992). 